
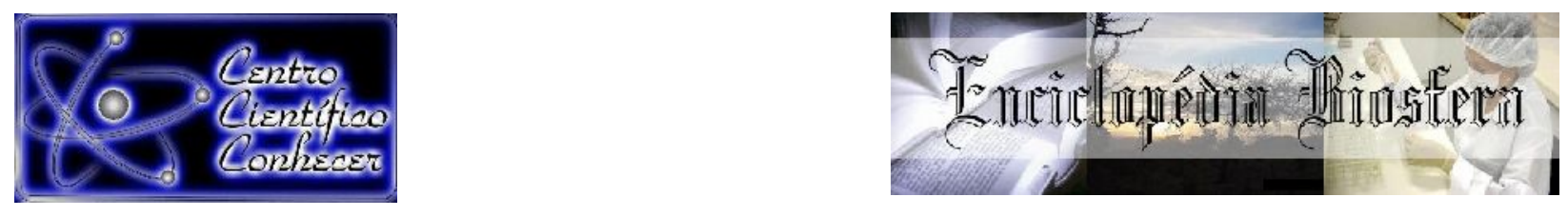

\title{
MENSURAÇÃO DA EFICIÊNCIA NA EDUCAÇÃO DOS MUNICÍPIOS GOIANOS: UMA ANÁLISE A PARTIR DO IDEB E DOS GASTOS NA EDUCAÇÃO BÁSICA
}

Márcio do Carmo Boareto ${ }^{1}$; Sara da Costa Fernandes²; Euclides Fernandes dos Reis $^{3}$; Dênia Aparecida de Amorim ${ }^{4}$, Vagner Rosalem ${ }^{5}$.

${ }^{1}$ Mestrando em Gestão Organizacional pela Universidade Federal de Catalão (UFCAT), Catalão-GO, Brasil (marcioboareto@gmail.com).

${ }^{2}$ Mestre em Gestão Organizacional pela Universidade Federal de Goiás (UFCAT), Catalão-GO, Brasil.

${ }^{3}$ Mestrando em Gestão Organizacional pela Universidade Federal de Catalão (UFCAT), Catalão, Goiás.

${ }^{4}$ Prof. Especialista na Fundação Carmelitana Mário Palmério (FUCAMP), Monte Carmelo-MG, Brasil.

${ }^{5}$ Prof. Doutor na Universidade Federal de Catalão (UFCAT), Catalão-GO, Brasil.

Recebido em: 06/04/2019 - Aprovado em: 10/06/2019 - Publicado em: 30/06/2019 DOI: 10.18677/EnciBio_2019A168

\section{RESUMO}

O objetivo deste trabalho foi mensurar o nível de eficiência da educação básica dos municípios goianos nos anos de 2013, 2015 e 2017 a partir do índice de desenvolvimento da educação básica (IDEB) e dos gastos em educação. Com a utilização do DEA (Data Envelopment Analysis), pelo método VRS (Variable Return Scale) foi calculado o nível de eficiência dos municípios a partir dos indicadores do IDEB (anos iniciais e anos finais), como outputs e do total de docentes, número de estabelecimentos de ensino, total investido e despesas com professor por aluno como inputs, resultando num conjunto de municípios eficientes e ineficientes e comparou-se os resultados de ambos em cada ano. Pela análise foi possível verificar que a média entre os valores dos municípios eficientes eram significativamente diferentes dos municípios ineficientes. Os resultados demonstraram que os municípios eficientes foram os que mais investiram nos alunos, com menos gastos com professores, menores quantidades de docentes e de estabelecimentos de ensino na educação básica.

PALAVRAS-CHAVE: data envelopment analysis, educação básica; gastos em educação. 


\title{
MEASUREMENT OF EFFICIENCY IN THE EDUCATION ON GOIÁS MUNICIPALITIES: AN ANALYSIS FROM IDEB AND EXPENDITURE IN BASIC EDUCATION
}

\begin{abstract}
The objective of this study was to measure the efficiency level of basic education in the municipalities of Goiás in the years 2013, 2015 and 2017, based on the index of development of basic education (IDEB) and expenditures on education. Using the DEA (Data Envelopment Analysis), using the VRS method (Variable Return Scale), the level of efficiency of the municipalities was calculated from the indicators of the IDEB (initial and final years), as outputs and the total number of teachers, number of educational establishments, total invested and expenses with teacher per student as inputs, resulting in a set of efficient and inefficient municipalities and compared the results of both in each year. By the analysis it was possible to verify that the average between the values of the effective municipalities were significantly different from the inefficient municipalities. The results showed that efficient municipalities were the ones that invested most in the students, with less on teachers' expenses, lower amounts of teachers and educational establishments in basic education.
\end{abstract}

KEYWORDS: data envelopment analysis, basic education; expenditures on education.

\section{INTRODUÇÃO}

A educação básica é o início do ciclo de aprendizagem dos futuros cidadãos e carece de atenção, pois, é a partir da educação que o indivíduo desenvolve suas habilidades de discernimento e crescimento intelectual. Segundo Lopes et al., (2018), fazem parte da Educação Básica a Educação Infantil, o Ensino Fundamental e o Ensino Médio, visto que o conceito de Educação Básica também engloba outras modalidades de ensino como a Educação de Jovens e Adultos, a Educação Especial e o Ensino Profissional. Assim, a educação infantil é a fase inicial da educação básica e objetiva estimular e incentivar o desenvolvimento da criança, enquanto que - Ensino Fundamental é um período mais extenso e tem como função o aperfeiçoamento na alfabetização, com foco no desenvolvimento físico, cognitivo, social, psicológico e intelectual do aluno. Já o ensino médio tem o intuito de consolidar e aprofundar os conhecimentos adquiridos na educação fundamental, desenvolvendo a compreensão e o domínio de fundamentos científicos e tecnológicos (LOPES et al., 2018).

No Brasil, a educação básica é um direito constitucional. Conforme Martin e Oliveira (2019), em dezembro de 1948, a Organização das Nações Unidas (ONU), naquela época composta por 58 Estados membros, inclusive o Brasil, instituiu a Declaração Universal dos Direitos Humanos (DUDH), na qual definiu os direitos básicos do ser humano, sendo listados direitos básicos para a promoção de uma vida digna para os habitantes do mundo, independente de nacionalidade, cor, sexo, raça, orientação sexual, política e religiosa, e que foi um passo para garantir a todos o direito, além de outras coisas, a educação. No Brasil, em 2003, o governo federal iniciou a formulação do Plano Nacional de Educação em Direitos Humanos (PNEDH), visando garantir a educação à pessoa com deficiência, a profissionalização de jovens e adultos, a erradicação do analfabetismo e a valorização dos educadores (MARTIN; OLIVEIRA, 2019).

O PNEDH iniciou-se com a criação do Comitê Nacional de Educação em Direitos Humanos (CNEDH), composto por especialistas, representantes da 
sociedade civil, instituições públicas, privadas e organismos internacionais. O Plano atua em cinco linhas da educação, sendo: a básica, a superior, a não formal, a dos profissionais de justiça e segurança e por último, a educação de mídia. A educação básica, a primeira e principal vertente, compreende que a educação em direitos humanos vai além de uma aprendizagem cognitiva, incluindo o desenvolvimento social e emocional. Assim, o PNEDH apresenta princípios norteadores para a educação básica, como a função de desenvolver uma cultura de direitos humanos em todos os espaços sociais. A escola deve assegurar que os objetivos e práticas a serem abordadas no ensino atendam aos valores e princípios da educação em Direitos Humanos (MARTIN; OLIVEIRA, 2019).

No intuito de conduzir políticas públicas a favor da qualidade da educação, foi criado no ano de 2007 o Índice de Desenvolvimento da Educação Básica (IDEB), reunindo em um único indicador o rendimento escolar e o desempenho da educação básica, estabelecendo como meta para o Brasil, até o ano de 2022, um índice de 6,0, fixado pelo Termo de Adesão ao Compromisso "Todos pela Educação" do Ministério da Educação, sendo esta meta comparada a de países desenvolvidos (INEP, 2019).

Conforme Lopes et al., (2018), por tratar-se de um país subdesenvolvido, o Brasil ainda possui dificuldade em diversos fatores como sociopolíticos, culturais, econômicos, e nas políticas educacionais, na qual o grande problema da educação está no financiamento, pois este é originado dos impostos arrecadados, e a porcentagem destes destinada a educação é insuficiente e muitas vezes utilizada de maneira indevida. As escolas públicas devem fornecer ensino de qualidade e uma boa estrutura, e isto só é possível através de investimentos financeiros.

Neste contexto, o Estado de Goiás conseguiu atingir as metas estabelecidas pelo Ministério da Educação em relação ao IDEB nos últimos três anos apenas no ensino fundamental, mas no ensino médio ficou aquém para os anos de 2015 e 2017. Com os 246 municípios distribuídos por regiões diferentes às dificuldades enfrentadas, a escassez de recursos, tanto humanos quanto financeiros, torna-se essencial uma análise mais aprofundada do desempenho dos municípios em relação à educação básica (INEP, 2019).

Esse estudo apresenta sua relevância apoiado na atual situação das escolas brasileiras, visto que os investimentos financeiros são insuficientes, além de utilizados de maneira indevida, sendo mal distribuídos ou desviados com ineficiências significativas (WORLD BANK, 2017). Portanto, a mensuração da eficiência dos gastos em educação básica dos municípios goianos, com base nos dados do IDEB, comparando-se informações dos anos iniciais e finais, apresenta importância teórica a nível regional e nacional, pois, pode servir de referência para outras análises na educação básica, além de direcionar a atenção para as escolas com índices insuficientes.

Desta forma o objetivo deste estudo foi mensurar a eficiência dos gastos em educação básica dos municípios goianos a partir dos dados do IDEB (anos iniciais e finais), número de estabelecimentos de ensino, total de docentes, investimento por aluno e gasto com professor por aluno na educação básica nos anos de 2013, 2015 e 2017.

\section{MATERIAL E MÉTODOS}

Tratou-se de um estudo descritivo e quantitativo objetivando a descrição das características e as relações entre as variáveis de estudo (GIL, 2007). Para composição da amostra dos municípios goianos foram retirados os municípios de 
Anhanguera, Baliza e Moiporá para aos anos de 2013 e 2015 e Anhanguera e Baliza para o ano de 2017, já que estes municípios não divulgaram as informações referentes ao IDEB, portanto a amostra foi composta por 243 municípios nos dois primeiros anos e 244 municípios no último ano.

Para análise do nível de eficiência dos municípios foram utilizados os valores referentes às variáveis descrita no quadro 2 , somente da rede pública da educação básica de ensino do Estado de Goiás. Para o cálculo da eficiência utilizou-se o modelo DEA-VRS (DATA ENVELOPMENT ANALYSIS - VARIABLE RETURN SCALE) com orientação a output (saída), ou seja, desejou-se maximizar as saídas mantendo as entradas inalteradas e permitindo que os retornos de escala fossem variáveis, pois este método é uma alternativa e um complemento aos métodos de análise de tendência central e permite calcular o nível de eficiência com base em dados reais (LINS; MEZA, 2000) conforme quadro 1.

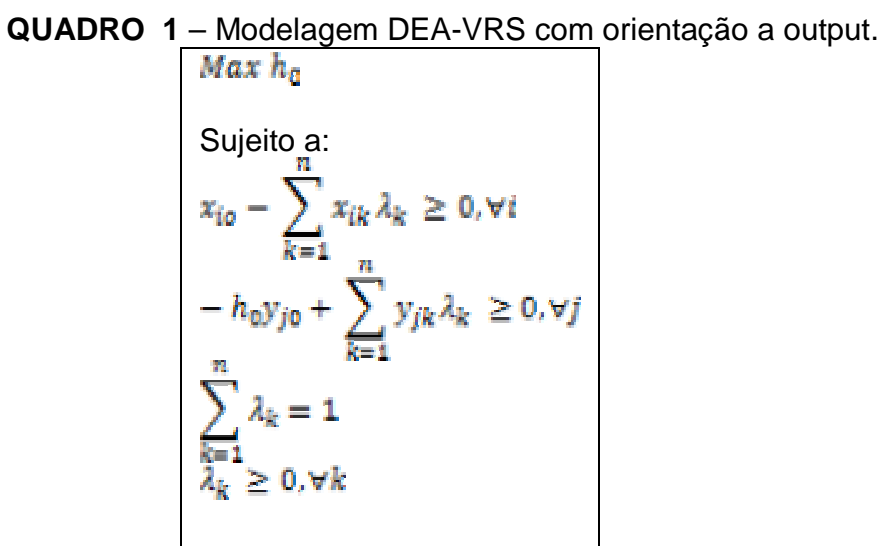

Os dados referentes aos valores do IDEB foram retirados do portal do Instituto Nacional de Estudos e Pesquisas Educacionais Anísio Teixeira (INEP, 2019) nos três últimos anos de divulgação, já que os dados são divulgados a cada dois anos. As demais informações sobre o número de estabelecimentos de ensino, total de docentes, investimento por aluno e gasto com professores por aluno da educação básica foram retirados do Sistema de Informações sobre Orçamentos Públicos em Educação (SIOPE, 2019).

QUADRO 2- Variáveis Utilizadas no estudo - DEA

\begin{tabular}{c|l|c}
\hline IDEB_I & Índice de Desenvolvimento da Educação Básica nos Anos Iniciais 1a ao 5a ano & Output \\
\hline IDEB_F & Índice de Desenvolvimento da Educação Básica nos Anos Finais 5 ao 9a ano & Output \\
\hline ESTAB & Total de Estabelecimentos de Ensino da Educação Básica por Município & Input \\
\hline DOCENTES & Número de Docentes da Educação Básica por Município & Input \\
\hline INVEST_ALUNO & Investimento Educacional por Aluno da Educação Básica (4.8) & Input \\
\hline GAST_PROF & Despesas com Professores por Aluno da Educação Básica (4.10) & Input \\
\hline
\end{tabular}

Fonte: Elaborado pelos Autores

Após o cálculo dos índices de eficiência de cada município separou-se os municípios em dois grupos, os eficientes e os ineficientes para verificar se as médias entre os valores eram estatisticamente diferentes nos dois grupos. Para proceder ao cálculo das diferenças das médias nos dois grupos verificou-se a normalidade de todas as variáveis pelo teste de Shapiro-Wilk, as variáveis IDEB_I $\left.\left.\left(S W_{2013}(243)=0,992 ; 0,178\right) / S W_{2015}(243)=0,993 ; 0,303\right) / S W_{2017}(244)=0,991 ; 0,137\right)$ 
e IDEB_F $\left.\quad\left(S W_{2013}(243)=0,993 ; \quad 0,369\right) \quad / \quad S W_{2015}(243)=0,991 ; \quad 0,164\right) \quad /$ $\left.S W_{2017}(244)=0,991 ; 0,139\right)$ apresentaram normalidade e as demais variáveis são significativamente não normais $(p<0,05)$, desta forma optou-se pelo teste $T$-Student para duas amostras independentes nas variáveis com distribuição normal e para as demais o teste de $U$ de Mann-Whitney para verificar se as diferenças entre as médias dos valores dos municípios eficientes e os ineficientes são estatisticamente diferentes (FIELD, 2009; MARÔCO, 2011).

O teste T-Student é indicado para testar se as médias de duas amostras independentes são ou não significativamente diferentes desde que possuam distribuição normal, e o teste de $U$ de Mann-Whitney também serve para testar as diferenças entre duas médias, mas não tem como pré-requisito que a distribuição seja normal, podendo ser utilizado como alternativa ao teste T-Student (MARÔCOO, 2011). O objetivo desta análise é comparar os valores médios encontrados entre os municípios eficientes dos ineficientes e se são estatisticamente diferentes, ou seja, os dispêndios efetuados pelos municípios na educação básica interferiram no nível de eficiência, já que, em média, os municípios eficientes possuíam menos estabelecimentos de ensino, docentes, gastos com professor, mas teve maior investimento educacional por aluno na educação básica.

\section{RESULTADOS E DISCUSSÃO}

A partir dos valores do nível de eficiência dos municípios, obtidos pelo modelo DEA-VRS, foi possível destacar os municípios que obtiveram valores de $100 \%$ de eficiência dos demais. Para o ano de 2013 foram analisados 243 municípios goianos e apenas 37 municípios tiveram valores de $100 \%$ conforme figura 1 . A média de eficiência total ficou em $87,17 \%$ para os municípios, sendo que o ano de 2013 apresentou menor nível de eficiência. Vale ressaltar que o município de Cavalcante teve o menor nível de eficiência, 65\%, com IDEB para os anos iniciais e finais de 4,4 e 3,6 respectivamente, bem abaixo da média dos municípios no período, já que o IDEB serve para acompanhamento da qualidade e importante condutor de políticas públicas em prol da educação básica no Brasil (INEP, 2019). 


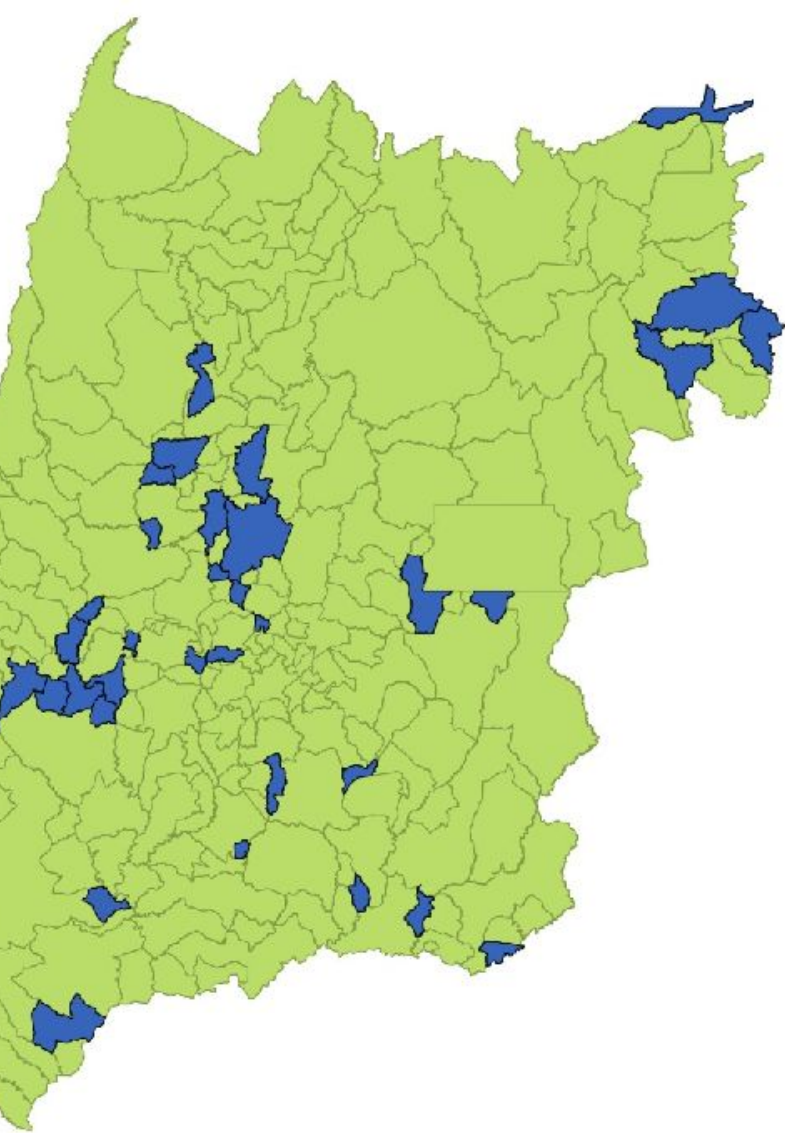

Guarinos

Itaguari

Ivolāndia

Jaraguá

Lagoa Santa

Mambaí

Marzagão

Maurilândia

Morro Agudo de Golás

Nova Aurora

Paranaiguara

Perolándia

Posse

Professor Jamil

Rubiataba

Santa Isabel

Santa Rosa de Goiás

Santo Antōnio do

Descoberto

Săo Joăo da Paraúna

Três Ranchos

Uruana

FIGURA 1 - Municípios eficientes e ineficientes (2013)

Fonte: Elaborado pelos autores (2019)

No ano de 2015 foram analisados 243 municípios dos quais 45 foram eficientes, conforme demonstrado na figura 2. Destaca-se que uma quantidade maior de municípios do sul do Estado obteve nível de eficiência de 100\% em relação ao ano anterior. A eficiência média para este ano ficou em $88,40 \%$, um pouco acima do ano anterior. Neste ano o município que apresentou o menor nível de eficiência foi Pilar de Goiás com 69,51\%, com valores de IDEB de 4,8 e 4,1 para os anos iniciais e finais respectivamente. 
Panamà

Paranaiguara

Porangatu

Santa Eárbara de Goiás Santa Earbara

FIGURA 2 - Municípios eficientes e ineficientes (2015)

Fonte: Elaborado pelos autores (2019)

No ano de 2017 foram analisados 244 municípios e destes 39 foram eficientes, com uma média de $89,53 \%$, ou seja, dos anos analisados este foi o que teve maior nível de eficiência total. O município de Mimoso de Goiás apresentou o menor nível com índice de 65,14\%, com valores do IDEB (iniciais e finais) de 4,8 e 3,7 respectivamente, bem abaixo da média dos municípios para o ano. Pelos resultados obtidos é possível identificar a limitação de cada município do Estado de Goiás e a localização dos eficientes, destacando que nos três anos a maior parte da eficiência concentrou-se nos municípios do centro do estado, demonstrando que a qualidade e eficiência na educação estão atreladas à forma de gestão dos recursos disponíveis (FERRI et al., 2017). 


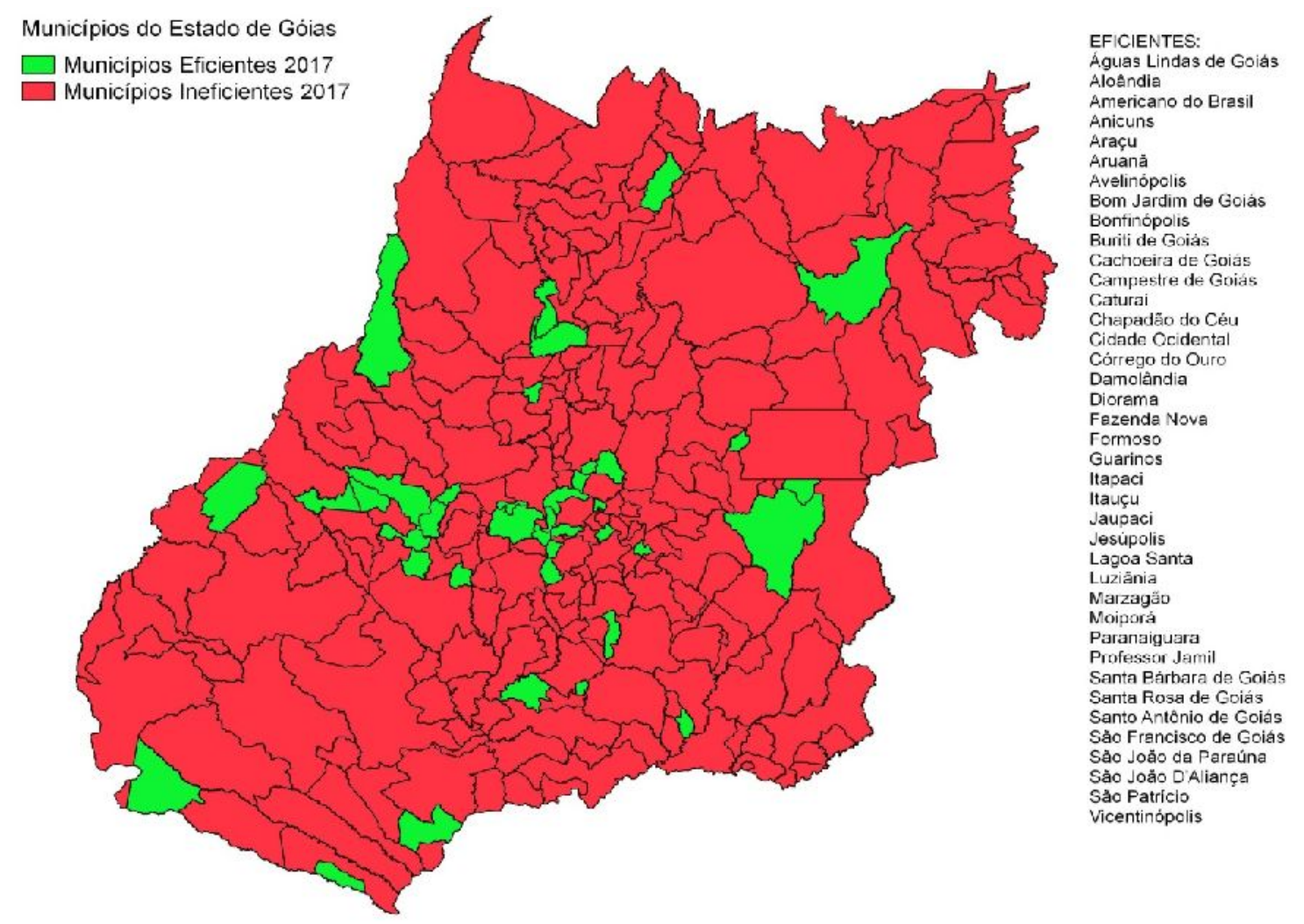

FIGURA 3 - Municípios eficientes e ineficientes (2017)

Fonte: Elaborado pelos autores (2019)

A partir da eficiência calculada pelo DEA-VRS foram classificados os municípios eficientes e os ineficientes dos anos 2013, 2015 e 2017. A eficiência média no período analisado foi de 88,37\%, 40 municípios eficientes e 203 ineficientes e o ano de 2017 apresentou o maior nível de eficiência (89,53\%) conforme tabela 1.

TABELA 1 - Eficiência x Municípios

\begin{tabular}{c|c|c|c}
\hline & EFICIÊNCIA & EFICIENTES & INEFICIENTES \\
\hline 2017 & $89,53 \%$ & 39 & 205 \\
2015 & $88,40 \%$ & 45 & 198 \\
2013 & $87,17 \%$ & 37 & 206 \\
\hline MÉDIA & $88,37 \%$ & 40 & 203 \\
\hline
\end{tabular}

Fonte: Elaborado a partir dos dados da pesquisa

A tabela 2 demonstra os valores médios dos municípios que foram eficientes, destacando que o gasto com professor foi menor que o investimento por aluno e a proporção de docentes por estabelecimento de ensino foi considerada elevada apresentando uma realidade bem diferente da encontrada nos municípios menos eficientes, indicando que há maior incidência de desperdícios na provisão da educação pública, necessitando de aprimoramento na gestão com a focalização na alocação dos recursos para maior economia e aumento do nível de eficiência da educação básica com a utilização de políticas adequadas (CASTRO et al., 2017). Tal fato revela também uma heterogeneidade na distribuição dos docentes nos municípios e com isso pode interferir na qualidade da avaliação do IDEB, ou seja, a 
discrepância na relação aluno/docente pode estar prejudicando no ensino/aprendizagem (GOMES, 2017).

TABELA 2 - Valores Médios Referentes aos Municípios Eficientes

\begin{tabular}{c|c|c|cccc|c|c}
\hline ANO & ESTAB & DOCENTES & \multicolumn{2}{|c|}{ INVEST_ALUNO } & GAST_PROF & IDEB_I & IDEB_F \\
\hline 2017 & 5 & 81 & $\mathrm{R} \$$ & $7.039,59$ & $\mathrm{R} \$$ & $4.460,85$ & 6,51 & 5,55 \\
2015 & 4 & 49 & $\mathrm{R} \$$ & $7.594,06$ & $\mathrm{R} \$$ & $3.756,29$ & 6,09 & 5,15 \\
2013 & 5 & 60 & $\mathrm{R} \$$ & $7.647,77$ & $\mathrm{R} \$$ & $3.925,93$ & 5,93 & 5,01 \\
\hline MÉDIA & 5 & 63 & $\mathrm{R} \$$ & $7.427,14$ & $\mathrm{R} \$$ & $4.047,69$ & 6,18 & 5,24 \\
\hline
\end{tabular}

Fonte: Elaborado a partir dos dados da pesquisa

A tabela 3 apresenta os valores dos municípios que foram ineficientes, e percebe-se que o número de estabelecimentos de ensino e o total de docentes apresentou o dobro do valor em relação aos municípios eficientes, já o investimento por aluno e o gasto com professor teve a mesma tendência dos eficientes, ou seja, investiu-se mais em aluno e menos em gasto com professor, mas a média deste ficou maior. Observa-se que não há um padrão de eficiência, devido principalmente a autonomia política e administrativa que os municípios brasileiros detêm, gerando possibilidades de diferentes formas de alocação dos recursos. Além disso, os municípios goianos apresentam elevado número de professores temporários, interferindo no vínculo com o aluno e com o próprio estabelecimento de ensino, atrapalhando na identificação de déficits educacionais dos alunos e problemas pedagógicos da própria escola, reduzindo a proposição de melhores soluções no ensino (GOMES, 2017; PENA et al., 2012a).

TABELA 3 - Valores Médios Referentes os Municípios Ineficientes

\begin{tabular}{cc|c|cc|cc|c|c}
\hline ANO & ESTAB & DOCENTES & \multicolumn{2}{|c|}{ INVEST_ALUNO } & GAST_PROF & IDEB_I & IDEB_F \\
\hline 2017 & 11 & 133 & $\mathrm{R} \$$ & $6.831,80$ & $\mathrm{R} \$$ & $4.649,52$ & 5,94 & 5,15 \\
2015 & 11 & 140 & $\mathrm{R} \$$ & $7.119,01$ & $\mathrm{R} \$$ & $4.334,38$ & 5,56 & 4,75 \\
2013 & 11 & 133 & $\mathrm{R} \$$ & $6.289,66$ & $\mathrm{R} \$$ & $3.745,37$ & 5,58 & 4,64 \\
\hline MÉDIA & 11 & 135 & $\mathrm{R} \$$ & $6.746,82$ & $\mathrm{R} \$$ & $4.243,09$ & 5,69 & 4,85 \\
\hline
\end{tabular}

Fonte: Elaborado a partir dos dados da pesquisa

Após a análise dos valores dos municípios que foram eficientes e ineficientes com valores médios distintos, buscou-se verificar se as médias nos dois grupos são significativamente diferentes, ou seja, se as variáveis realmente interferiram no cálculo da eficiência entre os dois grupos, já que o maior dispêndio de recursos, sem o respectivo endereçamento dos problemas de eficiência não garante melhores resultados e sim pode resultar em maiores desperdícios principalmente nos municípios goianos, onde a predominância de ineficiências na provisão da educação pública é elevada, além de que, resultados melhores poderiam ter sido obtidos com menos recursos (CASTRO et al., 2017; PENA et al., 2012b; SCHETTINI, 2018; WORLD BANK, 2017).

A tabela 4 demonstra o teste de significância para as diferenças entre as médias do IDEB nos dois grupos de municípios. Nos três anos analisados as diferenças entre as médias foram significativas, $p<0,05$, ou seja, existe diferença entre as médias do IDEB entre os municípios eficientes dos ineficientes, 
confirmando o modelo DEA-VRS, pois o modelo com orientação à saída (output) tende a evidenciar os municípios que obtiveram nota maior no IDEB.

TABELA 4 - Teste T-Student para os dados IDEB (Iniciais / Finais)

\begin{tabular}{cc|c|c|c|c|c|c|c|c|c|c|c}
\hline \multicolumn{4}{c}{2013} & \multicolumn{4}{c}{2015} \\
\hline & $\mathrm{gl}$ & $\mathrm{t}$ & sig & dif.md & $\mathrm{gl}$ & $\mathrm{t}$ & sig & dif.md & $\mathrm{gl}$ & $\mathrm{t}$ & sig & dif.md \\
\hline IDEB_I & 241 & $-2,88$ & 0,004 & $-0,34$ & 241 & $-4,69$ & 0,000 & $-0,53$ & 242 & $-5,14$ & 0,000 & 0,11 \\
IDEB_F & 241 & $-3,32$ & 0,002 & $-0,37$ & 241 & $-4,31$ & 0,000 & $-0,40$ & 242 & $-4,98$ & 0,000 & 0,08
\end{tabular}

Fonte: Elaborado a partir dos dados da pesquisa

A tabela 5 demonstra a análise das demais variáveis pelo teste de $U$ de Mann-Whitney para o ano de 2013 a fim de verificar se os valores médios são significativamente diferentes, ou seja, quais variáveis influenciaram no resultado da eficiência dos municípios, dando significantes as médias do número de estabelecimentos de ensino, total de docentes e o gasto com professor por aluno. Destaca-se o valor máximo investido por aluno pelo município de Aloândia $(\mathrm{R} \$$ 56.505) bem acima do valor médio apresentado no ano.

TABELA 5 - Teste $U$ de Mann-Whitney - 2013 entre Municípios Eficientes X Ineficientes

\begin{tabular}{|c|c|c|c|c|c|c|c|c|}
\hline & \multirow{2}{*}{ MIN } & \multirow{2}{*}{ MAX } & \multicolumn{3}{|c|}{ Percentis } & \multirow{2}{*}{$U$} & \multirow{2}{*}{ Z } & \multirow{2}{*}{ Sig } \\
\hline & & & 250. & $50^{\circ}$ & $75^{\circ}$ & & & \\
\hline ESTAB & 1 & 285 & 2 & 4 & 9 & 1955,50 & $-4,74$ & 0,000 \\
\hline DOCENTES & 2 & 5127 & 24 & 43 & 97 & 2201,00 & $-4,09$ & 0,000 \\
\hline INVEST_ALUNO & $\begin{array}{c}\mathrm{R} \$ \\
3.499\end{array}$ & $\begin{array}{c}\mathrm{R} \$ \\
56.505\end{array}$ & $\begin{array}{c}\mathrm{R} \$ \\
4.960 \\
\end{array}$ & $\begin{array}{c}\mathrm{R} \$ \\
5.879\end{array}$ & $\begin{array}{c}\mathrm{R} \$ \\
7.147\end{array}$ & 3497,00 & $-0,80$ & 0,425 \\
\hline GAST_PROF & $\begin{array}{c}\mathrm{R} \$ \\
1.351\end{array}$ & $\begin{array}{c}\mathrm{R} \$ \\
25.956\end{array}$ & $\begin{array}{c}\mathrm{R} \$ \\
2.906\end{array}$ & $\begin{array}{c}\mathrm{R} \$ \\
3.423\end{array}$ & $\begin{array}{c}\mathrm{R} \$ \\
4.256\end{array}$ & 2673,00 & $-2,89$ & 0,000 \\
\hline
\end{tabular}

Fonte: Elaborado a partir dos dados da pesquisa

A tabela 6 para o ano de 2015 confirmou os valores do ano anterior, destacando o valor máximo de investimento por aluno pelo município de Ivolândia $(\mathrm{R} \$ 37.520)$ e pelo valor máximo gasto por aluno com professor pelo município de Aloândia ( $R \$$ 9.761) bem abaixo do maior gasto no ano anterior pelo mesmo município.

TABELA 6- Teste $U$ de Mann-Whitney - 2015 entre Municípios Eficientes X Ineficientes

\begin{tabular}{|c|c|c|c|c|c|c|c|c|}
\hline & \multirow{2}{*}{ MIN } & \multirow{2}{*}{ MAX } & \multicolumn{3}{|c|}{ Percentis } & \multirow{2}{*}{ U } & \multirow{2}{*}{ Z } & \multirow{2}{*}{ Sig } \\
\hline & & & 250. & $50^{\circ}$ & $75^{\circ}$ & & & \\
\hline ESTAB & 1 & 300 & 2 & 4 & 9 & 2314,00 & $-5,06$ & 0,000 \\
\hline DOCENTES & 4 & 5103 & 24 & 44 & 94 & 2837,50 & $-3,80$ & 0,000 \\
\hline INVEST_ALUNO & $\begin{array}{c}\mathrm{R} \$ \\
3.131\end{array}$ & $\begin{array}{c}\mathrm{R} \$ \\
37.520\end{array}$ & $\begin{array}{c}R \$ \\
5.538\end{array}$ & $\begin{array}{c}R \$ \\
6.632\end{array}$ & $\begin{array}{c}\mathrm{R} \$ \\
7.855\end{array}$ & 3903,00 & $-1,30$ & 0,195 \\
\hline GAST_PROF & $\begin{array}{c}\mathrm{R} \$ \\
1.428\end{array}$ & $\begin{array}{c}R \$ \\
9.761\end{array}$ & $\begin{array}{c}\mathrm{R} \$ \\
3.365\end{array}$ & $\begin{array}{c}\mathrm{R} \$ \\
4.021 \\
\end{array}$ & $\begin{array}{c}\mathrm{R} \$ \\
4.865 \\
\end{array}$ & 3019,00 & $-3,37$ & 0,001 \\
\hline
\end{tabular}

Fonte: Elaborado a partir dos dados da pesquisa 
A tabela 7 demonstra somente a diferença entre as médias dos valores de estabelecimentos de ensino e número de docentes da educação básica dos municípios, ou seja, somente estes dois resultados teve significância para o nível de eficiência. Outro ponto a ser destacado é o número máximo de docentes que foi informado pelo município de Goiânia (4842) bem inferior aos valores máximos apresentados nos anos anteriores (5127-5103).

TABELA 7- Teste U de Mann-Whitney - 2017 entre Municípios Eficientes X Ineficientes

\begin{tabular}{|c|c|c|c|c|c|c|c|c|}
\hline & \multirow{2}{*}{ MIN } & \multirow{2}{*}{ MAX } & \multicolumn{3}{|c|}{ Percentis } & \multirow{2}{*}{ U } & \multirow{2}{*}{ Z } & \multirow{2}{*}{ Sig } \\
\hline & & & 250. & $50^{\circ}$ & $75^{\circ}$ & & & \\
\hline ESTAB & 1 & 303 & 2 & 4 & 10 & 1969,50 & $-5,05$ & 0,000 \\
\hline DOCENTES & 4 & 4842 & 24 & 45 & 101 & 2347,50 & $-4,08$ & 0,000 \\
\hline INVEST_ALUNO & $\begin{array}{c}R \$ \\
4.090\end{array}$ & $\begin{array}{c}\mathrm{R} \$ \\
23.769\end{array}$ & $\begin{array}{c}R \$ \\
5.543\end{array}$ & $\begin{array}{c}R \$ \\
6.510\end{array}$ & $\begin{array}{c}\mathrm{R} \$ \\
7.577\end{array}$ & 3838,00 & $-0,39$ & 0,693 \\
\hline GAST_PROF & $\begin{array}{c}R \$ \\
1.932\end{array}$ & $\begin{array}{c}\mathrm{R} \$ \\
12.477\end{array}$ & $\begin{array}{c}\mathrm{R} \$ \\
3.617\end{array}$ & $\begin{array}{c}R \$ \\
4.427\end{array}$ & $\begin{array}{c}\mathrm{R} \$ \\
5.115\end{array}$ & 3350,00 & $-1,60$ & 0,109 \\
\hline
\end{tabular}

Fonte: Elaborado a partir dos dados da pesquisa

Pela análise apresentada percebe-se que os municípios que tiveram maiores investimentos com alunos e menores gastos com professor foram os mais eficientes, pelo teste estatístico comprovou-se que os valores médios utilizados com estabelecimentos de ensino, número de docentes e gasto com professor foram significativamente diferentes nos anos de 2013 e 2015, já no ano de 2017 apenas os números com estabelecimentos de ensino e docentes foram diferentes, pois a qualidade do gasto público tem grande importância e maiores gastos em educação não significa necessariamente aumento da eficiência, já que pode afetar a boa gestão dos recursos públicos (KAKIHARA et al., 2016).

Apesar de o modelo DEA-VRS ser mais benevolente, ou seja, atribuir para um maior número de casos analisados o status de eficiente, foi possível determinar, com o uso conjunto de outros métodos estatísticos, o que realmente ocasionou a diferença entre os eficientes dos ineficientes. Destaca-se que nos municípios ineficientes a proporção de docentes para cada estabelecimento de ensino foi menor, podendo indicar uma sobrecarga de trabalho para os professores, ou nível de esforço docente elevado em relação à atuação em mais de uma escola, turnos e etapas de ensino, podendo ocasionar também baixas proporções de docentes formados na disciplina que atua, ou seja, a utilização de mão de obra sem a devida qualificação (GOMES, 2017; VITELLI et al., 2018).

Vale destacar que a educação básica no Brasil apresenta ineficiências significativas, principalmente na baixa relação aluno/professor e desperdícios na execução dos gastos educacionais. Municípios mais ricos, com alta taxa de receita por aluno, tendem a serem menos eficientes, pois devido a regras constitucionais, muitos são obrigados a efetuar gastos em itens que não necessariamente ampliam o aprendizado e que haveria uma economia de até 1\% no PIB (Produto Interno Bruto) se tivesse uma melhora no desempenho dos serviços de educação básica brasileira (WORLD BANK, 2017).

A universalização da educação básica no Brasil tem permitido acesso a todos os cidadãos, mas ainda carece de melhorias na qualidade do ensino, pois apesar de aumento do gasto educacional, ainda persiste níveis consideráveis de ineficiências, 
pois políticas de valorização dos profissionais da educação, melhorias na formação dos professores e na infraestrutura das escolas e uma gestão eficiente dos recursos poderia melhorar o desempenho da educação como um todo (MARTIN; OLIVEIRA, 2019).

\section{CONCLUSÕES}

O presente estudo mensurou o nível de eficiência da educação básica nas escolas públicas dos municípios goianos a partir dos resultados do IDEB e dos gastos em educação identificando que os municípios eficientes foram os que mais investiram nos alunos, com menores gastos com professores, menores quantidades de docentes e de estabelecimentos de ensino.

A qualidade na educação e o processo de ensino e aprendizagem dependem de vários fatores e não somente do aporte de recursos financeiros, pois o nível de eficiência da educação depende de ações públicas que consigam efetivamente entender seus problemas, suas dimensões e sua real necessidade e o diagnóstico da atual situação do ensino público possibilita uma gestão eficiente para a melhoria de seu desempenho.

No cenário atual onde diversos municípios veem suas receitas diminuírem e os diversos gastos aumentarem, com exigências legais e demandas cada vez mais específicas por parte da população, uma gestão eficiente dos recursos pode fazer toda a diferença e contribuir para a melhoria do serviço prestado pelo ente público.

Como limitação o estudo apresenta poucas variáveis que influenciaram a eficiência da educação básica nos municípios e apenas três anos e não se pode concluir que o fator preponderante para o nível de eficiência foi o total de estabelecimentos de ensino e do número de docentes apresentados pelos municípios, apesar de que a diferença destes valores entre os dois grupos foi significante.

Como sugestão para futuras pesquisas indica-se o estudo de mais variáveis em relação ao desempenho dos alunos no IDEB, e os motivos da diferença entre a quantidade de estabelecimentos de ensino, número de docentes e o total gasto em educação básica e se esses fatores estão associados ao menor nível de eficiência na educação básica dos municípios goianos.

Vale destacar que a análise proposta permitiu mensurar os valores gastos com professores e o total investido por aluno na educação básica em relação do desempenho no IDEB dos municípios goianos, também permitiu verificar a estrutura oferecida pelos municípios em relação ao total de estabelecimentos de ensino e a quantidade de professores da educação básica.

\section{REFERÊNCIAS}

CASTRO, C. R. DE M.; SOUZA, G. DA S. E; TANNURI-PIANTO, M. E. Gastos em educação: mais recursos sem gestão? Economia Aplicada, v. 21, n. 2, p. 285-309, 2017. Disponível em: http://www.revistas.usp.br/ecoa/article/view/135145. DOI: 10.11606/1413-8050/ea154258.

FERRI, C. M.; PEREIRA, R. S.; VIEIRA, S. F. A.; VISENTIN, I. C.. A eficiência na alocação dos recursos de escolas municipais de Luziânia/GO: um estudo a partir da análise envoltória de dados (DEA). Anais do Congresso Brasileiro de Custos ABC, v. 0, n. 0, 14 nov. 2017. Disponível em: https://anaiscbc.emnuvens.com.br/anais/article/view/4308. 
FIELD, A. Descobrindo a estatística usando o SPSS - Tradução Lorí Viali. 2. ed. Porto Alegre: Bookman - Artmed, 2009.

GIL, A. C. Como elaborar projetos de pesquisa. 4. ed. São Paulo: Atlas, 2007.

GOMES, R. R. Análise do Censo Escolar da Educação Básica 2016 - Perfil e Características dos Docentes do Estado de Goiás. Instituto Mauro Borges. Disponível em: <http://www.imb.go.gov.br/files/docs/releases/censo-escolareducacao-basica/censo-escolar-educacao-basica-2016.pdf>. Acesso em: 30 abr. 2019.

INEP. Instituto Nacional de Estudos e Pesquisas Educacionais Anísio Teixeira. Disponível em: <http://portal.inep.gov.br/web/guest/sinopses-estatisticas-daeducacao-basica>. Acesso em: 22 jan. 2019.

KAKIHARA, A. A. S. B. DE S.; SILVA, V. DE S. DA; JUNIOR, J. H. P. Análise da Eficiência do Gasto Público em Educação Fundamental em oito Diretorias de Ensino de São Paulo. Anais do XLVIII SBPO Simpósio Brasileiro de Pesquisa Operacional, p. 643-654, set. 2016. Disponível em: http://www.din.uem.br/sbpo/sbpo2016/pdf/156512.pdf. Acesso em: 01 fev. 2019.

LINS, M. P. E.; MEZA, L. A. Análise Envoltória de Dados e perspectivas de integração no ambiente de apoio à decisão. Rio de Janeiro-RJ: COPPE/UFRJ, 2000.

LOPES, B. G.; SILVA, E.; RIBEIRO, G.; PESSOA, M. K.; CARVALHO, P. S. Políticas Públicas de Financiamento da Educação Básica. Encontro Internacional de Gestão, Desenvolvimento e Inovação (EIGEDIN), v. 2, n. 1, 6 dez. 2018. Disponível em: http://seer.ufms.br/index.php/EIGEDIN/article/view/6280. Acesso em: 08 mar. 2019.

MARÔCO, J. Análise Estatística com o SPSS Statistics. 5. ed. Pero Pinheiro: Report Number, Lda., 2011.

MARTIN, J. A. P.; OLIVEIRA, E. A. A Declaração Universal dos Direitos Humanos e a Educação Brasileira. Revista Eletrônica da Educação, v. 2, n. 1, p. 35-46, 27 fev. $2019 . \quad$ Disponível em: http://portal.fundacaojau.edu.br:8077/journal/index.php/revista_educacao/article/view /57. Acesso em: 08 mar. 2019.

PENA, C. R.; ALBUQUERQUE, P. H.; DAHER, C. E. Dinâmica da produtividade e eficiência dos gastos na educação dos municípios goianos. RAC-Revista de Administração Contemporânea, v. 16, n. 6, p. 845-865, nov. 2012a. Disponível em: $\quad$ http://www.scielo.br/scielo.php?script=sci_abstract\&pid=S141565552012000600006\&lng=es\&nrm=iso\&tlng=pt. $\quad$ DOI: $\quad 10.1590 / S 1415-$ 65552012000600006 .

PENA, C. R.; ALBUQUERQUE, P. H. M.; MARCIO, C. J. A eficiência dos gastos públicos em educação: evidências georreferenciadas nos municípios goianos. Economia Aplicada, v. 16, n. 3, p. 421-443, 2012b. Disponível em: http://www.scielo.br/scielo.php?script=sci_arttext\&pid=S1413- 
$80502012000300004 \&$ Ing=pt\&nrm=iso\&tlng=en.

DOI:

$10.1590 / S 1413-$

80502012000300004 .

SCHETTINI, B. P. Avaliação da eficiência técnica dos municípios brasileiros na educação pública. Pesquisa e Planejamento Econômico, v. 48, n. 1, abr. 2018. Disponível em: http://ppe.ipea.gov.br/index.php/ppe/article/view/1720/126.

SIOPE. Sistema de Informações sobre Orçamentos Públicos em Educação. Disponível em: $<$ https://www.fnde.gov.br/siope/indicadoresFinanceirosEEducacionais.do?acao=ATU ALIZAR\&anoPaginacao $=-$

$1 \&$ paginacao $=\& p a g=$ result\&cod_uf $=52 \&$ municipios $=120001>$. Acesso em: 22 jan. 2019.

VITELLI, R. F.; FRITSCH, R.; CORSETTI, B. Indicadores educacionais na avaliação da educação básica e possíveis impactos em escolas de Ensino Médio no município de Porto Alegre, Rio Grande do Sul. Revista Brasileira de Educação, v. 23, n. 230065, 2018. Disponível em: http://dx.doi.org/10.1590/S1413-24782018230065. DOI: $10.1590 /$ S1413-24782018230065.

WORLD BANK. A fair adjustment: efficiency and equity of public spending in Brazil : Volume I: síntese (Portuguese). Washington, D.C.: World Bank Group., 1 nov. $2017 . \quad$ Disponível em: <http://documents.worldbank.org/curated/en/884871511196609355/Volume-Isíntese>. Acesso em: 30 abr. 2019. 